\title{
Comment on Ricci Collineations for type B warped space-times
}

\author{
M Tsamparlis*and P. S. Apostolopoulos*
}

June 17, 2021

\begin{abstract}
We present two counter examples to the paper by Carot et al. in Gen. Rel. Grav. (1997). 291223 and show that the results obtained are correct but not general.
\end{abstract}

KEY WORDS: Warped space-times: Ricci collineations

Suppose $\left(M_{1}, h_{1}\left(x^{A}\right)\right)$ and $\left(M_{2}, h_{2}\left(x^{\alpha}\right)\right)$ are a pair of Riemannian manifolds with co-ordinate functions $x^{A}(A, B, \ldots=1,2)$ and $x^{\alpha}(\alpha, \beta, \ldots=3,4)$ respectively. Let $\Phi\left(x^{C}\right)$ be a real valued function on $M_{1}$ and $M=M_{1} \times M_{2}$ be the product manifold (type B warped space-time) with metric 11]:

$$
d s^{2}=h_{A B}\left(x^{C}\right) d x^{A} d x^{B}+\Phi^{2}\left(x^{C}\right) h_{\alpha \beta}\left(x^{\gamma}\right) d x^{\alpha} d x^{\beta} .
$$

A vector field $\mathbf{X}$ in $M$ can be decomposed uniquely in "horizontal" and "vertical" components as follows:

$$
X^{a}=X_{1}^{A}\left(x^{b}\right) \delta_{A}^{a}+X_{2}^{\alpha}\left(x^{b}\right) \delta_{\alpha}^{a}
$$

where $a, b, \ldots=1,2,3,4$.

In a recent paper [2] (referred from now on as CNP) Carot et al. have considered the problem of determining all Ricci collineations (RCs) of type B warped space-times and have come to the following conclusion:

The horizontal component $\mathbf{X}_{2}$ of a proper $R C \mathbf{X}$ in a warped type $B$ space-time is either a Homothetic Vector Field (HVF) of $\left(M_{2}, h_{2}\right)$ and $\mathbf{X}$ is given by:

$$
\mathbf{X}=X_{1}^{A}\left(x^{a}\right) \partial_{A}+C^{I}\left(x^{B}\right) \boldsymbol{\zeta}_{I}
$$

$C^{I}$ and $X_{1}^{A}$ being functions of their arguments to be determined from the relations

$$
\begin{gathered}
R_{A B, D} X_{1}^{D}+R_{A D} X_{1, B}^{D}+R_{D B} X_{1, A}^{D}=0 \\
R_{A D} X_{1, \alpha}^{D}+F h_{\alpha \beta} X_{2, A}^{\beta}=0
\end{gathered}
$$

and $\left\{\boldsymbol{\zeta}_{I}\right\}$ (with $\left.I \leq 4\right)$ form a basis of the homothetic algebra of $\left(M_{2}, h_{2}\right)$ or

$\mathbf{X}_{2}$ it is a proper Special Conformal Killing Vector (SCKV) of $\left(M_{2}, h_{2}\right)$, this being possible only when $\left(M_{2}, h_{2}\right)$ is flat, in which case $X$ is given by:

${ }^{*}$ Department of Physics, Section of Astronomy-Astrophysics-Mechanics, University of Athens, ZOGRAFOS 15783, ATHENS, GREECE. E-mails:papost@cc.uoa.gr, mtsampa@cc.uoa.gr 


$$
\mathbf{X}=\left[P^{A}\left(x^{B}\right) x+Q^{A}\left(x^{B}\right)\right] \partial_{A}+\left[\frac{A_{0}}{2}\left(x^{2}-y^{2}\right)-D_{0} y+L\left(x^{B}\right)\right] \partial_{x}+\left(A_{0} x y+D_{0} x\right) \partial_{y}
$$

where the functions specifying $\mathbf{X}$ must satisfy (4), (5) and $x, y$ are co-ordinate functions in $\left(M_{2}, h_{2}\right)$.

Unfortunately the conclusion above is not the solution to the problem considered, in the sense that it does not give all (smooth) RCs of a type B warped space-time. We show this by giving the following two counter examples.

Counter example 1

$\overline{\text { Consider a globally }}\{2+2\}$ decomposable space-time i.e. $\Phi\left(x^{C}\right)=1$. It is always possible to choose co-ordinates $x^{a}=\{t, x, y, z\}$ in which the metric is written:

$$
d s^{2}=f^{2}\left(x^{C}\right)\left(-d t^{2}+d x^{2}\right)+f^{\prime 2}\left(x^{\gamma}\right)\left(d y^{2}+d z^{2}\right)
$$

where the functions $f, f^{\prime}$ are smooth functions of their arguments.

Consider the vector $\mathbf{X}=\partial_{t}+\partial_{y}$ and define the functions $f, f^{\prime}$ to be:

$$
\begin{aligned}
f & =e^{\frac{a_{1} t^{2}}{2}+b_{1} t+c_{1}} \\
f^{\prime} & =e^{\frac{a_{2} y^{2}}{2}+b_{2} y+c_{2}}
\end{aligned}
$$

It is easy to show that for this choice of $f, f^{\prime}$ the curvature scalars $R_{1}=2 a_{1} e^{-a_{1} t^{2}-2 b_{1} t-2 c_{1}}$, $R_{2}=-2 a_{2} e^{-a_{2} y^{2}-2 b_{2} y-2 c_{2}}$ do not vanish and furthermore $\mathcal{L}_{\mathbf{X}} R_{a b}=0, \mathcal{L}_{\mathbf{X}} g_{a b} \neq 2 \psi(\mathbf{X}) g_{a b}$ and $\mathcal{L}_{\mathbf{X}} R_{b c d}^{a} \neq 0$ so that $\mathbf{X}$ is a proper (smooth) RC of the $2+2$ metric $g_{a b}$. Taking the Lie derivative of the 2-metrics with respect to the projections $\mathbf{X}_{1}=\partial_{t}, \mathbf{X}_{2}=\partial_{y}$ of $\mathbf{X}$ in the 2-spaces we find:

$$
\begin{aligned}
\mathcal{L}_{\mathbf{X}_{1}} h_{A B} & =2\left(a_{1} t+b_{1}\right) h_{A B}=2 \psi_{1}\left(\mathbf{X}_{1}\right) h_{A B} \\
\mathcal{L}_{\mathbf{X}_{2}} h_{\alpha \beta} & =2\left(a_{2} y+b_{2}\right) h_{\alpha \beta}=2 \psi_{2}\left(\mathbf{X}_{2}\right) h_{\alpha \beta}
\end{aligned}
$$

which show that $\mathbf{X}_{1}, \mathbf{X}_{2}$ are proper $\mathrm{CKV}$ s of the 2 -spaces $\left(M_{1}, h_{1}\right)$ and $\left(M_{2}, h_{2}\right)$ respectively contrary to the conclusions of CNP. Furthermore it is easy to show that $\mathbf{X}_{1}, \mathbf{X}_{2}$ are proper Ricci collineations of the corresponding 2-metrics. [It is well known [3] that in a $2+2$ decomposable space-time the sum $\mathbf{X}=\mathbf{X}_{1}+\mathbf{X}_{2}$ of Ricci collineations $\mathbf{X}_{1}, \mathbf{X}_{2}$ of the 2-spaces $\left(M_{1}, h_{1}\right)$ and $\left(M_{2}, h_{2}\right)$ respectively, defines a Ricci collineation for the whole space-time.]

Counter example 2

In the $2+2$ decomposable space-time given above (and the same functions $f, f^{\prime}$ ) consider the vector field $\mathbf{X}=z \partial_{t}+\partial_{y}+t \partial_{z}$. It is easy to show that $\mathcal{L}_{\mathbf{X}} R_{a b}=0$ provided $a_{1}=-a_{2}$. Furthermore $\mathcal{L}_{\mathbf{X}} g_{a b} \neq 2 \psi(\mathbf{X}) g_{a b}$ and $\mathcal{L}_{\mathbf{X}} R_{b c d}^{a} \neq 0$. The projection $\mathbf{X}_{1}=z \partial_{t}$ is a proper CKV for the Lorentzian 2-space with conformal factor $\psi\left(\mathbf{X}_{1}\right)=z\left(b_{1}-a_{2} t\right)$ and the projection $\mathbf{X}_{2}=$ $\partial_{y}+t \partial_{z}$ is a proper CKV of the Euclidean 2-space with conformal factor $\psi\left(\mathbf{X}_{2}\right)=a_{2} y+b_{2}$ where both 2-spaces are not flat. Due to the dependence of $\mathbf{X}_{1}$ from $x^{4}$ and $\mathbf{X}_{2}$ from $x^{1}$, this counterexample also shows, that the Ricci collineations $\mathbf{X}_{1}, \mathbf{X}_{2}$ are not invariant under the isometries linking the timelike (spacelike) submanifolds of the decomposition [3].

As we have remarked the results obtained in CNP are correct but they are not general. This is due to the basic relation (17) of CNP which is not true for a 2-dimensional space. 
Indeed it can be proved that if $\mathbf{X}$ is a proper CKV of a $n$-dimensional space with metric $g_{a b}$ satisfying $\mathcal{L}_{\mathbf{X}} g_{a b}=2 \psi(\mathbf{X}) g_{a b}$ then the following identity holds:

$$
\mathcal{L}_{\mathbf{X}} R_{a b}=-(n-2) \psi_{; a b}-\left(\psi_{; c d} g^{c d}\right) g_{a b}
$$

where $R_{a b}$ is the Ricci tensor and a semicolon denotes covariant differentiation associated with the $n$-dimensional metric $g_{a b}$.

Hence for a proper CKV $\mathbf{X}_{2}$ of a 2 D metric $h_{\alpha \beta}$ i.e. $\mathcal{L}_{\mathbf{X}_{2}} h_{\alpha \beta}=2 \Psi\left(\mathbf{X}_{2}\right) h_{\alpha \beta}$ equation (9) implies:

$$
\mathcal{L}_{\mathbf{X}_{2}} R_{2 \alpha \beta}=-\left(\Psi_{\mid \rho \sigma} h^{\rho \sigma}\right) h_{\alpha \beta}
$$

where a stroke denotes covariant differentiation w.r.t. 2-metric $h_{\alpha \beta}$.

Thus, equation (21) of CNP does not follow from (17) but can be seen rather as an extra assumption which is consistent with equation (10).

To show this consider equation (10). Since for every 2 -space $R_{2 \alpha \beta}=\frac{R_{2}}{2} h_{\alpha \beta}$ equation (10) implies:

$$
\mathbf{X}_{2}\left(R_{2}\right)+2 \Psi R_{2}=-2\left(\Psi_{\mid \rho \sigma} h^{\rho \sigma}\right) .
$$

Contraction of equation (21) of CNP together with (11) gives $\mathbf{X}_{2}\left(R_{2}\right)+2 \Psi R_{2}=0$. Thus either $R_{2}=0$ or $\mathbf{X}_{2}\left(R_{2}\right)=-2 \Psi R_{2}$ where the later equation gives $\Psi_{, \alpha}=0$ i.e. $\mathbf{X}_{2}$ is a HVF for the metric $h_{\alpha \beta}$.

We conclude that the problem of determining the (proper and smooth) RCs of type B warped space-times is still open although a serious step towards its solution has been done in CNP.

\section{References}

[1] Carot, J., and da Costa, J., Class. Quantum Grav. (1993). 10, 461

[2] Carot, J., Nunez. L., and Percoco, U. Gen. Rel. Grav. (1997). 29, 1223

[3] Hall, G., S., Roy, I., and Vaz, E. G. L. R. (1996) Gen. Rel. Grav. 28, 299 